\title{
SCIENTIFIC REPORTS

\section{Identification of conserved and novel miRNAs responsive to heat stress in flowering Chinese cabbage using high-throughput sequencing}

\author{
Waqas Ahmed ${ }^{1,5}$, Yanshi Xia ${ }^{1,5}$, Hua Zhang ${ }^{2}$, Ronghua $\mathrm{Li}^{1}{ }^{1}$, Guihua Bai $\mathbb{1}^{3}$, \\ Kadambot H. M. Siddique $\mathbb{D}^{4}$ \& Peiguo Guo ${ }^{1 *}$
}

Plant microRNAs (miRNAs) are noncoding and endogenous key regulators that play significant functions in regulating plant responses to stress, and plant growth and development. Heat stress is a critical abiotic stress that reduces the yield and quality of flowering Chinese cabbage (Brassica campestris L. ssp. chinensis var. utilis Tsen et Lee). However, limited information is available on whether miRNAs are involved in the regulation of heat stress in B. campestris. A high-throughput sequencing approach was used to identify novel and conserved heat-responsive miRNAs in four small RNA libraries of flowering Chinese cabbage using leaves collected at $0 \mathrm{~h}, 1 \mathrm{~h}, 6 \mathrm{~h}$ and $12 \mathrm{~h}$ after a $38^{\circ} \mathrm{C}$ heat-stress treatment. The analysis identified 41 conserved miRNAs (belonging to 19 MIR families), of which MIR156, MIR159, MIR168, MIR171 and MIR1885 had the most abundant molecules. Prediction and evaluation of novel miRNAs using the unannotated reads resulted in 18 candidate miRNAs. Differential expression analysis showed that most of the identified miRNAs were downregulated in heat-treated groups. To better understand functional importance, bioinformatic analysis predicted 432 unique putative target miRNAs involved in cells, cell parts, catalytic activity, cellular processes and abiotic stress responses. Furthermore, the Kyoto Encyclopedia of Genes and Genomes maps of flowering Chinese cabbage identified the significant role of miRNAs in stress adaptation and stress tolerance, and in several mitogen-activated protein kinases signaling pathways including cell death. This work presents a comprehensive study of the miRNAs for understanding the regulatory mechanisms and their participation in the heat stress of flowering Chinese cabbage.

Brassica vegetable crops-including cabbage (red, green, etc.), brussels sprouts, broccoli, kale and cauliflowerbelong to the Brassicaceae family, all of which are cultivated and consumed throughout the world. Brassica species are morphologically and phytochemically diverse. Some Brassica species accumulate secondary metabolites such as phenolics and glucosinolates that are beneficial to human health. Flowering Chinese cabbage (Brassica campestris L. ssp. chinensis var. utilis Tsen et Lee) is one of the most commonly cultivated vegetable crops in the southern part of China ${ }^{1}$. Demand for flowering Chinese cabbage is increasing in China as it is reasonably priced and has high nutritional value, soluble fiber and vitamin C. However, due to genetic and adverse environmental stresses, the quality and productivity of flowering Chinese cabbage have declined ${ }^{2}$.

Heat stress is a major environmental factor that negatively affects physiological processes, reproduction, adaptation and crop development, which in turn reduce yield ${ }^{3,4}$. Global climate analysis predicted that high temperature is becoming more frequent and has the potential to affect crop yields in the near future ${ }^{5}$. Flowering Chinese cabbage is a cryophilic vegetable crop that has been planted year-round in southern China since its domestication several hundred years ago. It often suffers from high-temperature stress in summer ${ }^{6}$, which not only influences

${ }^{1}$ International Crop Research Center for Stress Resistance, College of Life Sciences, Guangzhou University, Guangzhou, 510006, China. ${ }^{2}$ Guangzhou Academy of Agricultural Sciences, Guangzhou, 510308, China. ${ }^{3}$ United States Department of Agriculture - Agricultural Research Service, Hard Winter Wheat Genetics Research Unit, Manhattan, Kansas, 66506, United States of America. ${ }^{4}$ The UWA Institute of Agriculture and School of Agriculture \& Environment, The University of Western Australia, LB 5005, Perth, WA, 6001, Australia. ${ }^{5}$ These authors contributed equally: Waqas Ahmed and YanshiXia. *email: guopg@gzhu.edu.cn 
plant growth and performance but results in yield reductions and even death ${ }^{7}$. Flowering Chinese cabbage genotypes can have obvious premature leaf senility, fine flowering stalks, significantly reduced photosynthetic capacity, and high electrolytic leakage and malondialdehyde content under high-temperature condition ${ }^{8,9}$.

Heat-stress tolerance in vegetable crops is a complex phenomenon involving various metabolic and biochemical processes, such as gene expression and translation, accumulation of compatible solutes, membrane lipid unsaturation, protein stability and antioxidant activity ${ }^{10}$. Plant noncoding RNAs play a significant role in the response to various abiotic and biotic stresses and are categorized into classes including microRNAs (miRNAs 21-24 nucleotides in length), circular RNAs (circRNAs), long noncoding RNAs (lncRNAs) and small interfering RNAs (siRNAs) ${ }^{11}$, which use various molecular mechanisms to perform their important functions, ranging from modulation of RNA stability, translation, post-transcriptional and transcriptional regulation of gene expression $^{12,13}$. Most of the identified miRNAs come from intergenic regions, but some conserved miRNAs have been derived from introns ${ }^{14}$. Several studies have reported various miRNAs involved in controlling various functions in Arabidopsis plants in response to heat stress ${ }^{15}$, miRNAs and their targets may play critical roles in stress resistance, including over-oxidation, phosphate starvation, polarity formation and morphogenesis, and hormone biosynthesis and signaling ${ }^{16,17}$. Recently, degradome analysis and high-throughput sequencing identified numerous miRNAs that might have essential functions in the heat-stress response of plants ${ }^{18-20}$. Using genomic and sequencing technologies, several miRNAs and their potential targets that play key roles in heat stress have been extensively studied in model plant species of various agricultural and vegetable crops ${ }^{21}$. Further studies have explored miRNA functions, including functional analysis of non-conserved and conserved miRNAs, their specific differential expressions and the quantification of the effects of miRNAs on their targets, which have highlighted their involvement in complex miRNA-mediated regulatory networks that play an essential role in controlling heat responses and tolerance ${ }^{22-24}$. Therefore, identification of the molecular mechanisms of heat tolerance of flowering Chinese cabbage is becoming an important research topic that will facilitate genetic improvement and production of this crop.

In our previous study, we reported express sequence tag-simple sequence repeat (EST-SSR) markers derived from RNA-seq of flowering Chinese cabbage after heat treatment. Those markers are valuable resources for genetic diversity analysis ${ }^{1}$. The identification of novel and conserved miRNAs that are responsive to heat stress offers an opportunity to better understand the molecular mechanisms of heat stress in flowering Chinese cabbage. To the best of our knowledge, no studies have identified heat-responsive miRNAs in flowering Chinese cabbage. This study aimed to identify novel miRNAs involved in heat stress, their differential expression, putative targets, and miRNA-related regulatory pathways linked to heat stress in flowering Chinese cabbage.

\section{Results}

Effect of heat stress on plant growth and antioxidant enzyme activity. High temperature had a marked impact on plant growth and development. Plant leaves showed wilting symptoms after exposure to high temperatures. The slight leave wilting was observed in heat treated group at $1 \mathrm{~h}$ of exposure to $38^{\circ} \mathrm{C}$, and then became very prominent at $6 \mathrm{~h}$ and $12 \mathrm{~h}$ (Fig. 1A), indicating that high temperature exereted severe water loss and inhibited growth of flowering Chinese cabbage. The activity of catalase (CAT) was significantly higher in leaves at $1 \mathrm{~h}, 6 \mathrm{~h}$ and $12 \mathrm{~h}$ than that in the $0 \mathrm{~h}$ control plants (Fig. 1B), with a maximum increase in CAT activity observed at $6 \mathrm{~h}$ of heat stress. In contrast, superoxide dismutase (SOD) and peroxidase (POD) activities in heat treated plants increased at $1 \mathrm{~h}, 6 \mathrm{~h}$ and $12 \mathrm{~h}$ compared to $0 \mathrm{~h}$ control plants (Fig. 1C,D). The findings showed that heat stress significantly increased CAT, SOD and POD activities in flowering Chinese cabbage.

sRNA sequencing in response to heat stress. Sequencing four libraries of flowering Chinese cabbage genotype Youlv 501 collected at $0 \mathrm{~h}, 1 \mathrm{~h}, 6 \mathrm{~h}$ and $12 \mathrm{~h}$ after heat stress generated 28,194,816, 29,746,062, $28,002,965$ and 29,049,979 raw reads, respectively. After removing reads with low-quality sequences-those without a $3^{\prime}$ primer or insert tag, shorter than $18 \mathrm{nt}$ and $5^{\prime}$ contaminants-26,293,810 (93.26\%), 27,726,336 (93.21\%), $25,883,914(92.43 \%)$ and $26,644,471(91.72 \%)$ clean reads remained for tissue collected $0 \mathrm{~h}, 1 \mathrm{~h}, 6 \mathrm{~h}$ and $12 \mathrm{~h}$ after heat stress, respectively (Table 1).

Subsequently, any sRNA reads that might belong to small nucleolar RNA (snoRNA), known rRNA, small nuclear RNA (snRNA), tRNA and unannotated reads were excluded (Table S1), leaving 20,775,702, 12,345,347, $12,704,323$ and 14,705,931 mappable small RNA sequences in the $0 \mathrm{~h}, 1 \mathrm{~h}, 6 \mathrm{~h}$ and $12 \mathrm{~h}$ libraries, respectively, for further consideration. The sRNAs varied extensively in their length distribution in the four libraries, ranging from 15 to $30 \mathrm{nt}$ (Fig. 2), with most ranging from 21-24 nt. Details of the sRNA length distributions with read counts in each library are in Table S2.

Conserved miRNAs responsive to heat stress. To examine conserved miRNAs in flowering Chinese cabbage, the sRNAs were exposed to a Blastn search against conserved miRNAs in miRBase 17.0, following no more than three mismatches. The length distribution of conserved miRNAs mostly occurred from 21-24 nt. To ensure the reliability of the results, conserved miRNAs with read counts $<10$ were excluded. The search identified 41 mature miRNA sequences of conserved miRNAs (details in Tables S3), of which bra-miR398-5p, bra-miR160a-5p, bra-miR168a-3p, bra-miR167a, bra-miR172b-5p, bra-miR159a, bra-miR162-3p, bra-miR403-3p, bra-miR396-5p, bra-miR158-3p, bra-miR168b-5p, bra-miR171a, bra-miR164a, bra-miR171e, and bra-miR168a-5p had very high expression levels (Fig. 3). Nineteen MIR families were identified from the sequencing analysis, of which MIR168, MIR156, MIR159, MIR171 and MIR1885 were the most abundant (Table S3). 


\begin{tabular}{|l|l|l|l|l|l|l|l|}
\hline Sample & $\begin{array}{l}\text { Total raw } \\
\text { read count }\end{array}$ & $\begin{array}{l}\text { Low-quality } \\
\text { read count }\end{array}$ & $\begin{array}{l}\text { Invalid adapter } \\
\text { read count }\end{array}$ & $\begin{array}{l}\text { Poly-A read } \\
\text { count }\end{array}$ & $\begin{array}{l}\text { Short valid } \\
\text { length reads }\end{array}$ & $\begin{array}{l}\text { Total clean } \\
\text { read count }\end{array}$ & $\begin{array}{l}\text { Percentage of } \\
\text { clean reads (\%) }\end{array}$ \\
\hline $\begin{array}{l}0 \mathrm{~h} \\
\text { control) }\end{array}$ & $28,194,816$ & 476,790 & 395,757 & 775 & $1,027,684$ & $26,293,810$ & 93.26 \\
\hline $1 \mathrm{~h}$ & $29,746,062$ & 619,057 & 416,205 & 684 & 983,780 & $27,726,336$ & 93.21 \\
\hline $6 \mathrm{~h}$ & $28,002,965$ & 568,450 & 423,553 & 746 & $1,126,302$ & $25,883,914$ & 92.43 \\
\hline $12 \mathrm{~h}$ & $29,049,979$ & 486,853 & 442,446 & 726 & $1,475,483$ & $26,644,471$ & 91.72 \\
\hline
\end{tabular}

Table 1. Numbers of sequence reads with varied quality in the small RNA databases of flowering Chinese cabbage from four libraries.
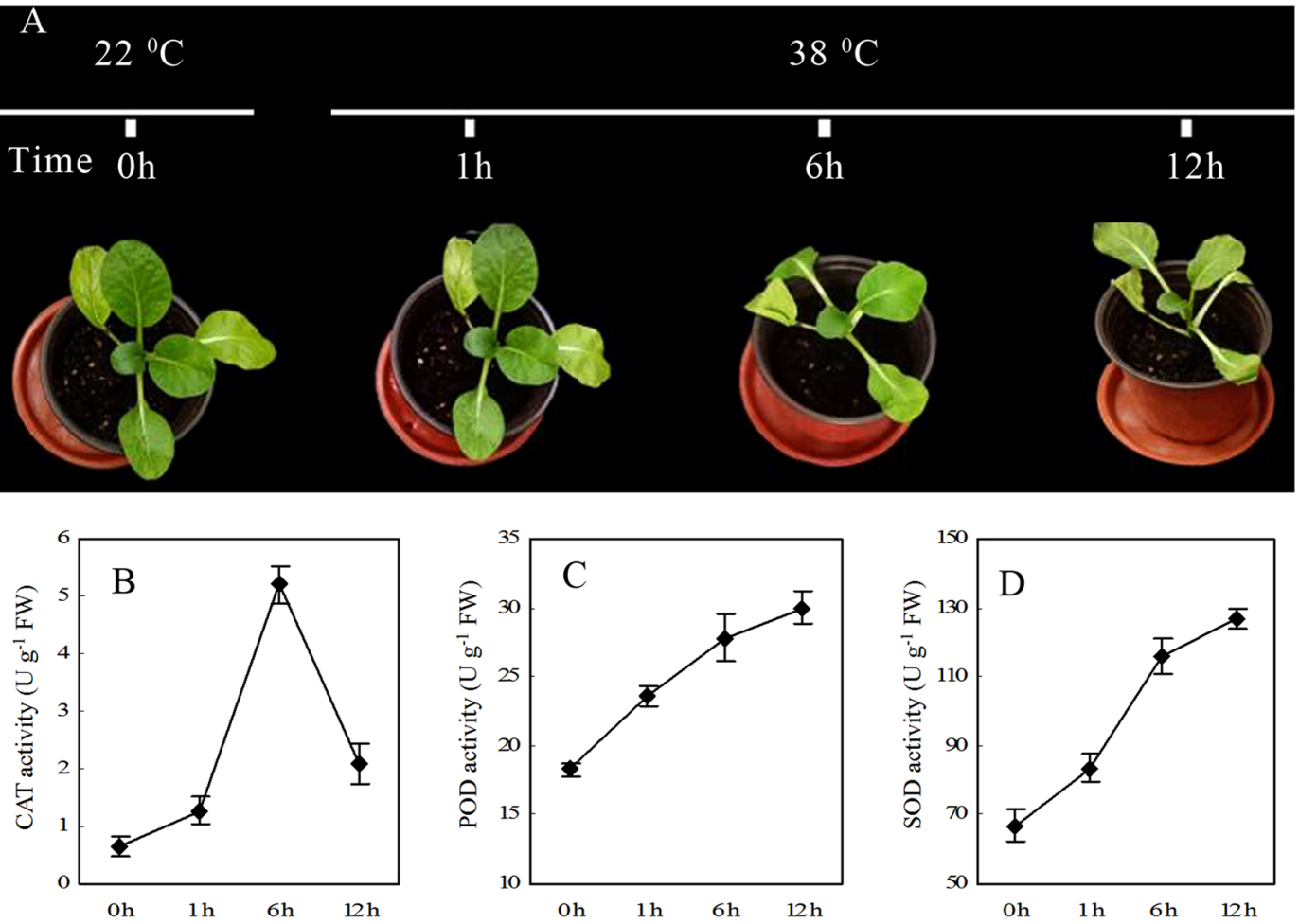

Figure 1. Physiological responses of flowering Chinese cabbage to heat stress.

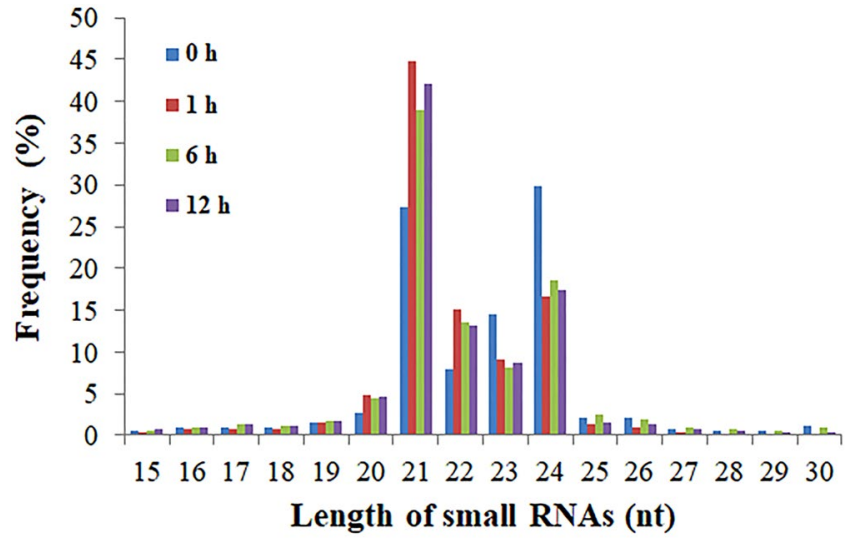

Figure 2. Sequence read length distribution of heat stress responsive small RNAs (sRNAs) in the libraries of flowering Chinese cabbage.

Novel miRNAs responsive to heat stress. To identify novel miRNAs involved in heat stress in flowering Chinese cabbage, small RNA mappable sequences were blasted against the Brassica database and then the miRBase to search for previously known miRNAs. The small RNAs not mapped as conserved miRNAs but exactly 


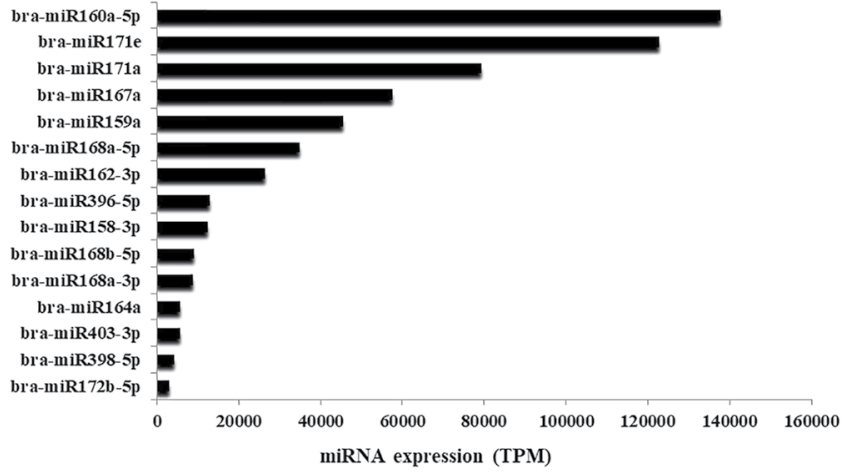

Figure 3. Top 15 abundantly expressed conserved miRNAs in flowering Chinese cabbage.

\begin{tabular}{|l|l|l|l|l|}
\hline Name & Location & Length & $\begin{array}{l}\text { Strand } \\
(+l-)\end{array}$ & Mature sequence \\
\hline Novel-mir001 & A01:13241536-13241782 & 23 & + & ATTTTGAGCATGTGGCTAGCTTT \\
\hline Novel-mir002 & A03:32323869-32324023 & 20 & + & ATTCATGCTTGACTACATCA \\
\hline Novel-mir003 & A03:2196941-2197178 & 24 & + & CGTAGACGCCGACGCAGTTTTAAT \\
\hline Novel-mir004 & A04:3008688-3008993 & 21 & + & ACAACACTCCCACAAGACGAG \\
\hline Novel-mir005 & A04:3841793-3841922 & 21 & + & ATGGCAGCCCATGTTAACATG \\
\hline Novel-mir006 & A05:11981503-11981733 & 22 & - & CGGTGGAAGGATTACGGGCCAG \\
\hline Novel-mir007 & A05:4940093-4940268 & 23 & + & CGTACCGCACCGCAGTTAACAGT \\
\hline Novel-mir008 & A05:662730-662965 & 24 & + & GTATCTTGGCCCCACAGAAGTGAT \\
\hline Novel-mir009 & A06:15296650-15296806 & 23 & + & TTGACGTAATCAGCCTCCAAATA \\
\hline Novel-mir010 & A06:12979559-12979722 & 23 & - & CCTCAGACCTGGATGTAGAAGTT \\
\hline Novel-mir011 & A06:16377258-16377447 & 25 & - & GCCCTATGAGATCCATTCCTGACGG \\
\hline Novel-mir012 & A08:9348257-9348369 & 25 & + & CTTCTCCAAGGCCTCACTTATCACC \\
\hline Novel-mir013 & A09:31314690-31314867 & 22 & + & TTTAACAGAGAGTAGAAACGGA \\
\hline Novel-mir014 & A09:24445902-24446047 & 24 & - & GGCGTCAGCGTCAATGAAAATAGT \\
\hline Novel-mir015 & A09:9886482-9886659 & 23 & + & TACCATACTGCCTAAGCCGAGTT \\
\hline Novel-mir016 & A09:33071308-33071419 & 22 & + & TATTGGCCTGGTTCACTCAGAT \\
\hline Novel-mir017 & A09:8151167-8151329 & 24 & + & GGTTTGAAGCGGTTTAGAACGATT \\
\hline Novel-mir018 & A10:19828692-19828861 & 24 & + & TCCGCCCCTGGGTTCGAGCCTTGG \\
\hline
\end{tabular}

Table 2. Sequences and chromosome locations of novel miRNAs from flowering Chinese cabbage under heat stress.

mapped to the genome sequence were considered as putative novel miRNAs. To enhance predictive accuracy in the identification of novel miRNAs, the miRNA/miRNA* criterion was the key consideration in our initial analysis. Using high-throughput sequencing, both candidate miRNA* and candidate miRNA sequences must be identified. miRNA and miRNA* must establish a duplex with nucleotides of $3^{\prime}$ overhangs in opposite stem-arms, mismatched bases must be $<3$, and the number of asymmetric bulges must be $\leq 1$. Also, miRNA precursors must have minimal folding energy indices (MFEI) and higher negative minimal folding energy (MFE) values to differentiate from other small RNAs. Eighteen potential novel miRNAs were identified from the four libraries (Table 2).

The mean MFE of predicted pre-miRNAs was $-38.27 \mathrm{kcal} / \mathrm{mol}$ (range -19.7 to $-207.4 \mathrm{kcal} / \mathrm{mol}$ ). The MFEI value ranged from 0.8 to 2.2, with an average of 1.4, indicating reliable key characteristic features of the predicted miRNAs. The length distribution of most of the novel identified miRNAs ranged from 21-24 nt. Novel-mir003, novel-mir009 and novel-mir016 were most abundantly expressed in the $1 \mathrm{~h}, 6 \mathrm{~h}$ and $12 \mathrm{~h}$ libraries, compared to $0 \mathrm{~h}$.

Identification of differentially expressed miRNAs. The conserved miRNA differential expression analysis revealed 16, 19 and 29 miRNAs with significant differential expression at $1 \mathrm{~h}, 6 \mathrm{~h}$ and $12 \mathrm{~h}$, respectively, relative to $0 \mathrm{~h}$ (Fig. 4), of which 11, 16 and 24 were downregulated and 5, 3 and 5 were upregulated, respectively (Table S4). Higher differential expression values occurred in three upregulated miRNAs (bra-miR5725, bra-miR5726 and bra-miR172c-3p) and four downregulated miRNAs (bra-miR390-3p, bra-miR156a-3p, bra-miR158-5p and bra-miR9557-3p).

The novel miRNA differential expression analysis identified 15, 18 and 18 miRNAs with key differential expression at $1 \mathrm{~h}, 6 \mathrm{~h}$ and $12 \mathrm{~h}$, respectively, relative to $0 \mathrm{~h}$ (Fig. 4), of which 12,12 and 11 were downregulated and 3, 6 and 6 miRNAs were upregulated, respectively. In general, the number of downregulated miRNAs was higher at all time points (Table S5). 

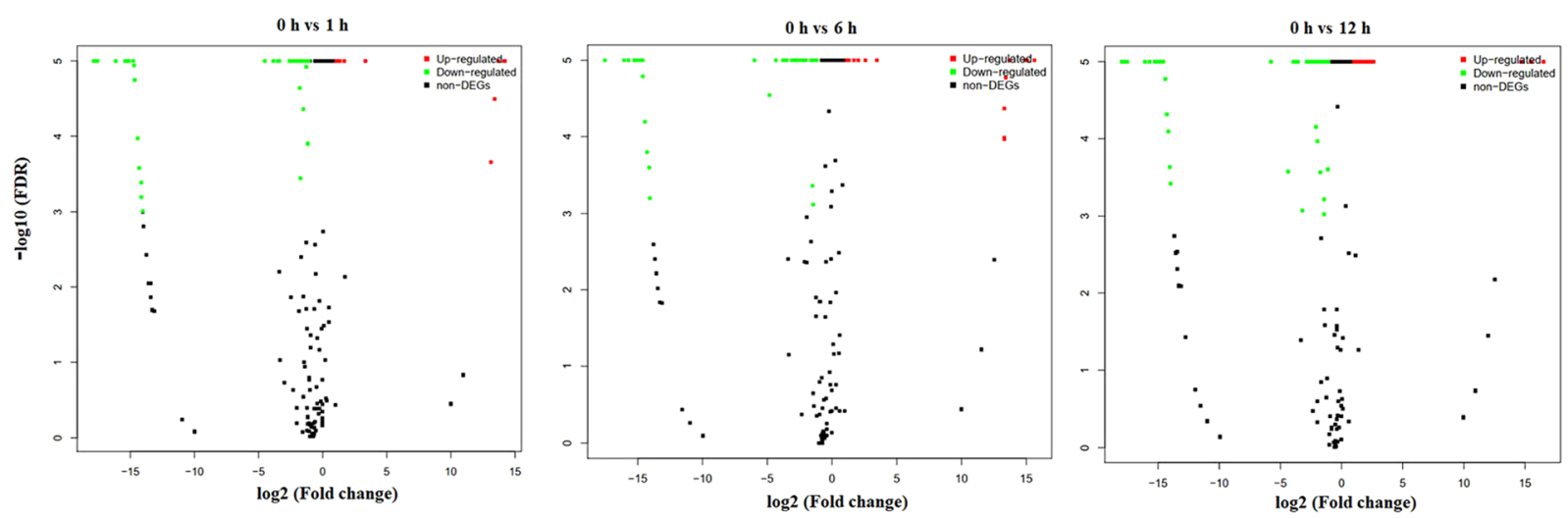

Figure 4. Volcano plots of differentially expressed heat-stress responsive miRNAs in flowering Chinese cabbage.

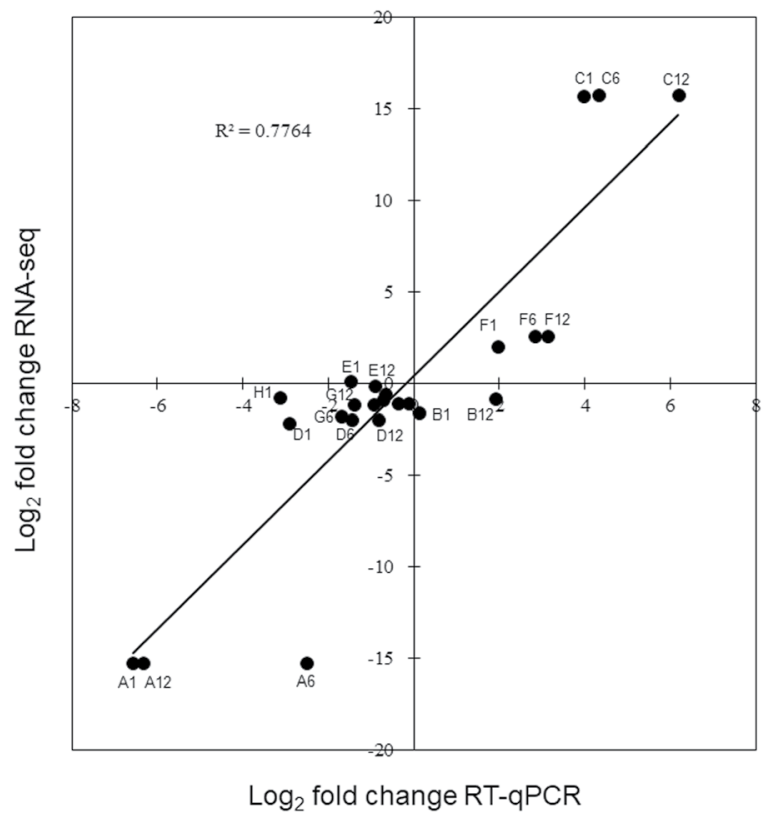

Figure 5. Validation of RNA-Seq results by RT-qPCR. Correlation between RNA-Seq and RT-qPCR data. The expression of eight miRNAs was validated using RT-qPCR. Letters A to $\mathrm{H}$ represent the miRNA names novelmir001, novel-mir007, novel-mir013, novel-mir017, bra-miR160a-3p, bra-miR159a, bra-miR158-5p and bramiR164a, respectively. Numbers 1,6 and 12 represent the corresponding time-points of $1 \mathrm{~h}, 6 \mathrm{~h}$ and $12 \mathrm{~h}$. The RT-qPCR $\log _{2}$-fold change values (X-axis) were plotted against RNA-seq data (Y-axis).

To validate the sequencing results, eight miRNAs (four novel and four conserved miRNAs), whose expression level covered both up- and down-regulated changes identified in the sequencing analysis, were randomly chosen for validation using RT-qPCR. The high correlation coefficient $\left(\mathrm{R}^{2}=0.776\right)$ between RNA-seq and RT-qPCR expression profiles is shown in Fig. 5, indicates that trends in expression level for the selected miRNAs from RT-qPCR were consistent (up-regulation or down-regulation) with those obtained by sequencing data, and thus directly confirms that sequencing analysis effectively-identified differentially expressed miRNAs of flowering Chinese cabbage with appropriate sensitivity.

Predicted target mRNA of the novel and conserved miRNAs. The identification of novel and conserved miRNA targets is essential for highlighting their potential functions in response to heat stress. We used TAPIR, TargetFinder and psRobot algorithms to screen and predict potential binding sites to annotate the mRNA targets for novel and conserved miRNAs response to heat stress. We identified 85 common spots between TAPIR and TargetFinder, 93 between TAPIR and psRobot, and 44 between TargetFinder and psRobot (Fig. 6), all of which were considered for further analysis.

KEGG pathway analysis of target genes. To investigate the molecular interaction networks and biological and cellular functions of miRNA responsive target genes, we used KEGG pathway analysis. The miRNA target genes were differentiated into groups using KEGG pathway analysis. The key targets for cellular processes 


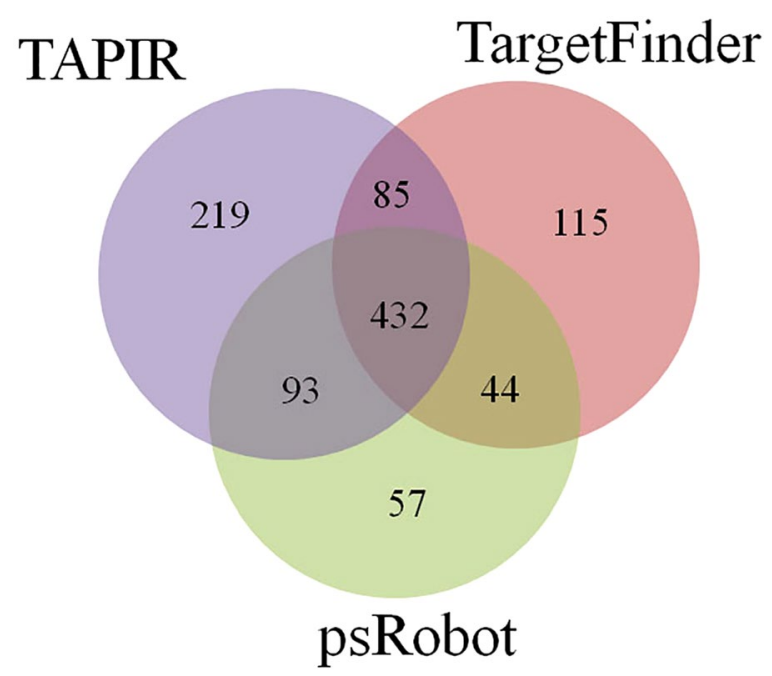

Figure 6. Comparison of numbers of predicted putative target mRNAs of heat-stress responsive miRNAs in the four flowering Chinese cabbage libraries among three prediction methods.

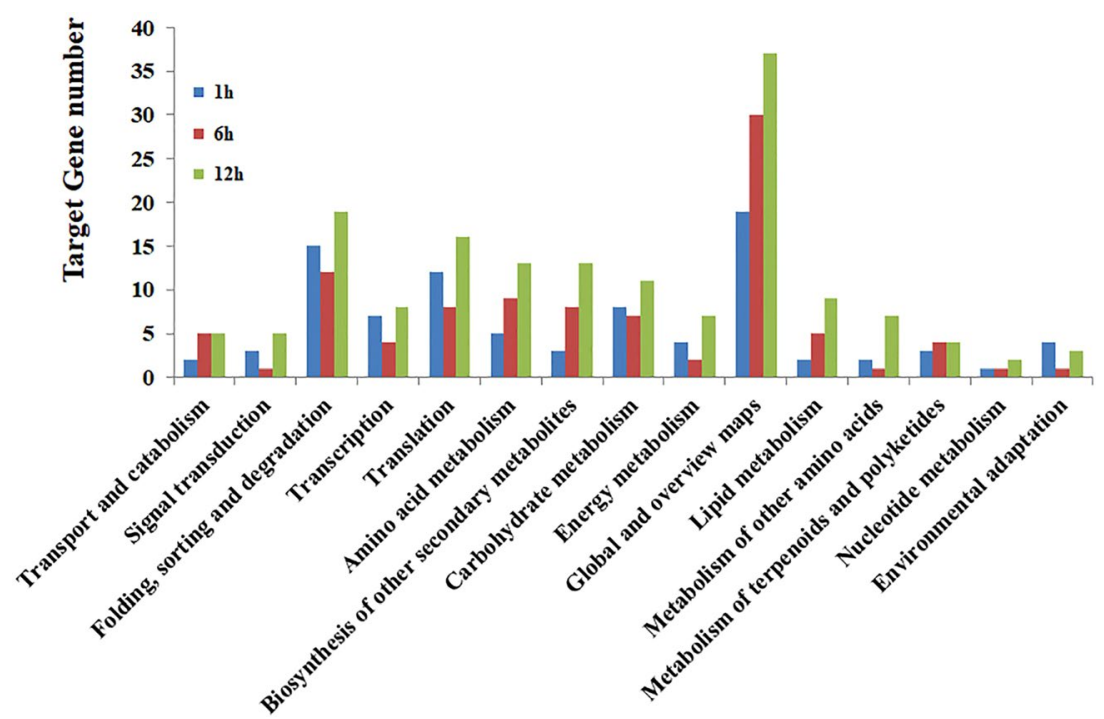

Figure 7. KEGG pathway analysis of target genes in heat-stress responsive miRNAs in the four flowering Chinese cabbage libraries.

and environmental information processing were signal transduction, transport and catabolism (Fig. 7), and for genetic information processing were folding, translation, transcription, sorting and degradation.

In the global and overview maps, amino acid and carbohydrate metabolism are important gene repertoire from metabolism while environmental adaptation is a significant gene from organismal systems, respectively, in the $1 \mathrm{~h}, 6 \mathrm{~h}$ and $12 \mathrm{~h}$ treatment. Detailed information on the KEGG category gene repertoires in all three treatments are listed in Table S6.

The KEGG pathway analysis also identified miRNA-regulated pathways from the predicted genes targeted and revealed that target genes were enriched for stress adaptation, stress tolerance, stress-tolerance response, and several MAPK signaling pathways, including cell death and early or late defense response for the pathogen (Fig. 8).

\section{Discussion}

Flowering Chinese cabbage is a significant vegetable crop that is commonly cultivated in southern China due to its favorable taste and nutrient value ${ }^{1}$. Climate changes and global warming cause cellular homeostasisthat depresses plant growth and development and increases disease infection ${ }^{25,26}$. Numerous stress-regulated miRNAs have been identified and functionally characterized in many vegetable crops under different abiotic and biotic stress conditions such as heavy metal homeostasis, salinity, heat, nutrient deficiency, drought, UV-B radiation and bacterial infection ${ }^{27-29}$. To investigate the molecular mechanisms underlying plant growth and development after heat stress, high-throughput sequencing and bioinformatic approaches have been used to identify novel and 


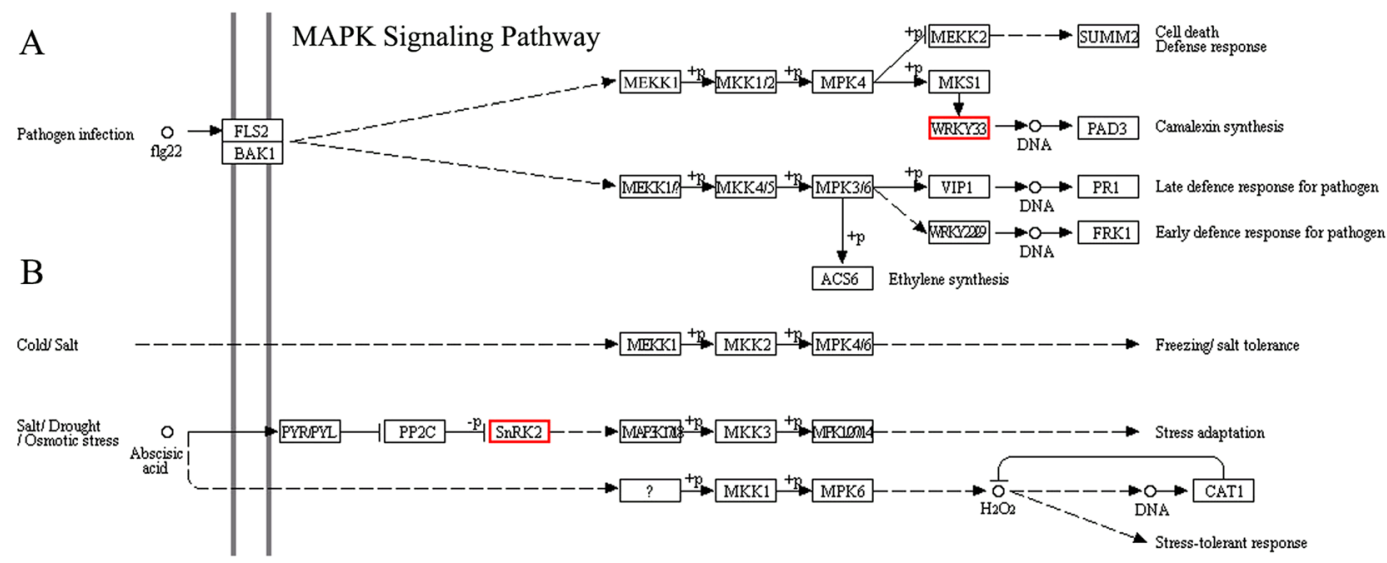

Figure 8. KEGG pathway map for MAPK signaling for flowering Chinese cabbage libraries. The KEGG pathway analysis also identified miRNA-regulated pathways from the predicted genes targeted ${ }^{54}$.

conserved miRNA in model organisms and important food crops ${ }^{30,31}$. To the best of our knowledge, only $66 B$. campestris miRNAs have been reported in the plant microRNA database ${ }^{32}$.

Yu et al.${ }^{33}$ identified 21 novel miRNAs from 19 miRNA families, of which four were responsive to heat in B. rapa, particularly bra-miR5718 and bra-miR1885b.3. Furthermore, high-throughput sequencing of $B$. rapa identified 125 novel and 221 conserved miRNAs that play a significant role in stress responses, metabolism, and growth and development ${ }^{34}$. Using a comparative genomics-based approach, 126 novel miRNAs have been identified in $B$. juncea that play significant roles in various biological processes including high temperature, drought and salinity, of which miR156, miR160 and miR164 were predicted to target SPL2-like, ARF17-like and a NAC (No Apical Meristem) domain-containing proteins, respectively ${ }^{35}$. In the current study, we identified 18 novel and 41 conserved miRNAs responsive to heat stress in flowering Chinese cabbage, and the most abundant sRNAs were 21 to $24 \mathrm{nt}$ long (Fig. 1), which is similar to those reported in Brassica rapa ${ }^{33}$ and B. oleracea ${ }^{36}$.

In our study, novel had less than 100 reads while most of the conserved miRNAs had more than 1000 reads. The highest numbers of reads were for bra-miR160a-5p (137,852 reads) and bra-miR171e (122,730 reads), which is similar to those detected in bra-miR159, which was almost near 45,000 32. Furthermore, miR157a, miR166a, miR167a, miR168c and miR172a had the most sequencing reads in Brassicaceae species ${ }^{37,38}$. In the current study, we found 19 MIR families with MIR168, MIR156, MIR159, MIR171 and MIR1885 the most abundant families (Table S3). In terms of MIR families, MIR158, MIR166, MIR167, MIR169, MIR391, MIR838, MIR824, MIR1140 and MIR1885 are most abundant ${ }^{11,27,38}$. In a study on $B$. oleracea, miR157a, miR166, miR167 and miR168c were the most abundant molecules and MIR166, MIR167 and MIR169 the largest miRNA families ${ }^{39}$. Similarly, the comparative genome-based computational analysis identified 193 potential miRNAs from genome survey sequences and expressed sequence tags in B. oleracea. MIR158, MIR156, MIR169 and MIR5021 were identified as the largest miRNAs families that have various functions in the regulation of different stimuli ${ }^{40}$.

miRNAs exhibit fluctuating expression patterns over time during expression profiling. In the present study, 16,19 , and 29 conserved miRNAs and 15, 18, 18 novel miRNAs exhibited differential expression at $1 \mathrm{~h}, 6 \mathrm{~h}$ and $12 \mathrm{~h}$, respectively, relative to $0 \mathrm{~h}$ (Tables S4 and S5). To verify the sequencing results, eight miRNAs-whose expression levels represent up- and down-regulated changes identified in the sequencing analysis-were randomly chosen for validation using RT-qPCR. The $\log _{2}$-fold change values and correlation coefficient $\left(\mathrm{R}^{2}=0.776\right)$ showed that expression profiles were consistent (up-/down-regulation) in the sequencing and RT-qPCR data. Similar studies have also validated the expression of several conserved and novel stress-responsive miRNAs using RT-qPCR ${ }^{35,36,41}$. Likewise, Jiang and colleagues identified 18 miRNAs with differential expression for pollen development in male sterile lines of $B$. campestris, relative to the male fertile line, of which three were downregulated and 15 were upregulated ${ }^{32}$. In recent years, numerous bioinformatics algorithms have been widely used for predicting plant miRNA targets, including TAPIR ${ }^{42}$, Target-align ${ }^{43}$ and psRNATarget ${ }^{44}$. In our study, the TAPIR, TargetFinder and psRobot algorithms identified 432 potential mRNA targets for novel and conserved miRNAs in B. campestris. Identification of target genes is the important and initial step to investigate the key function of miRNAs. Likewise, a computational approach and deep sequencing analysis identified 20 conserved miRNAs that exhibited significant differential expression between heat-sensitive and heat-tolerant genotypes under heat stress in B. olerace $^{39}$. Recently, different components in thermotolerance were identified that play key roles in the defense of oxidative damage ${ }^{36}$. The study revealed that the predicted miRNAs have an essential function in the regulation of target genes for shoot apical development, phase change, and hormone and energy metabolism, and the response to temperature stimulus ${ }^{36}$. Another study on B. oleracea predicted that miRNAs might target mRNAs encoding several potential proteins involved in the regulation of various functions, including abiotic stress response, oxidative phosphorylation, cell communication and hormone stimuli ${ }^{39}$. Several miRNAs have been identified as involved in lipid and fatty acid metabolism, including miR1134, miR156e-p3, miR838-p3, miR9563a-p3, miR159c, and miR9563b-p5 that could target WSD1, PlCD6, PDP, LACS9, ADSL, C0401, MFPA, OLEO3, ACO32 and GDL73 in Brassica napus ${ }^{45}$. Furthermore, Wang et al. (2012b) identified several miRNAs targets that participate in various functions, including plant development, signal transduction and stress response. 
In conclusion, we have presented the first comprehensive heat-responsive small RNA dataset for B. campestris L. ssp. chinensis var. utilis Tsen et Lee. Using next-generation sequencing and a biocomputational approach, we identified 18 novel and 41 conserved miRNAs. The putative target prediction determined the roles and functions of the identified miRNAs in flowering Chinese cabbage. The identified miRNA sequences, their differential expression, and predictive mRNA targets presented in the current study can be used to plan crop development approaches in flowering Chinese cabbage and related species. Since the full genome of flowering Chinese cabbage had not yet been reported, the entire set of miRNAs and its possible targets could not be identified. However, this study has delivered significant information on the stated molecules and their involvement in different functions.

\section{Materials and Methods \\ Plant materials, sample collection and total RNA extraction. Flowering Chinese cabbage (Brassica campestris L. ssp. chinensis var. utilis Tsen et Lee) genotype Youlv 501 was grown in a growth chamber at the experimental station of Guangzhou University set at $28 / 22^{\circ} \mathrm{C}$ for $14 / 10 \mathrm{~h}$ (day/night). Plants were moved into another growth chamber set at $38 / 29^{\circ} \mathrm{C}(14 / 10 \mathrm{~h})$ at the five-leaf stage for the heat-stress treatment. Samples were collected from the upper fully expanded leaves at four time-points $(0 \mathrm{~h}$ (control), $1 \mathrm{~h}, 6 \mathrm{~h}$ and $12 \mathrm{~h}$ after the start of the heat-stress treatment), flash-frozen in liquid nitrogen, and kept at $-80^{\circ} \mathrm{C}$ until further needed. Trizol RNA extraction kit (Invitrogen, USA) was used to isolate total RNA following the manufacturer's instructions.}

Determination of antioxidant enzyme activities. The activity of antioxidant enzymes (SOD, POD and CAT) were measured using assay kits (Nanjing Jiancheng Bioengineering Institute, China) following the manufacturers' instructions. Briefly, fresh leaves $(0.5 \mathrm{~g})$ were crushed with pestle and mortar and homogenized with $50 \mathrm{mM}$ phosphate buffer ( $\mathrm{pH} 7.0)$. The homogenate samples were centrifuged at $3,500 \mathrm{~g}$ for $10 \mathrm{~min}\left(4^{\circ} \mathrm{C}\right)$. Enzyme activities were determined with a spectrophotometer using the supernatants. All procedures were carried out at $0-4^{\circ} \mathrm{C}$.

Construction of sRNA libraries and sequencing. The RNA for each time point was isolated from five individual replicates and mixed in an equivalent proportion. The cDNA library was constructed using an Illumina TruSeq Small RNA Preparation Kit following the manufacturer's recommendations. In brief, (1) RNA $3^{\prime}$ - and RNA $5^{\prime}$-adapters were ligated to total RNA, (2) cDNA constructs were generated using reverse transcription after PCR, and (3) small cDNA fragments were isolated in different lengths (18-30 nt) using 6\% denaturing polyacrylamide gel electrophoresis. The purified cDNA library was subjected to next-generation sequencing using Illumina HiSeq technology at the Beijing Genomics Institute (BGI, Shenzhen, China) following the manufacturer's instruction for running the instrument. Raw sequencing was collected using Illumina's analysis software.

Bioinformatics analysis of sequencing data to identify novel and conserved miRNAs. Raw sequence reads were collected and analyzed after removing adapter sequences, oversized insertion tags, no-insert tags, $5^{\prime}$-primer contaminants, low-quality tags, poly-A tags and small tags (those without $3^{\prime}$ primers and sequences beyond 15-30 nucleotides). To analyze small RNA length distributions, the Bowtie 2 web program was used to map clean reads to the reference genome and other sRNA databases ${ }^{46}$. The unique sRNAs were aligned to known non-codingRNAs downloaded from the Rfam database http://www.sanger.ac.uk/science/tools/rfam/ with use of the NCBI BLASTN to discard tRNA, rRNA, snRNA, scRNA and snoRNA. Matched sequences were excluded for further analysis, unmatched sequences were subjected to evaluation of matched and mismatched sequences through the Brassica database (http://brassicadb.org/brad/). The miRNAs were considered potential conserved candidates if they had no more than three nucleotide of mismatches, while candidate novel miRNAs were reserved in unmatched sequences. Finally, miRBase software was used to predict novel miRNAs from unannotated clean sequences ${ }^{47}$.

Identification of differentially expressed miRNAs. The control and heat-stress treatments were compared to identify differential expression of miRNAs in flowering Chinese cabbage under heat stress. The levels of miRNA expression were normalized to transcripts per million in all libraries and converted to 0 to 0.01 to avoid calculation error. If the miRNA had normalized expression of $<1$ in all libraries, then it was excluded from further comparative analysis due to low expression. The normalization equation is:

Normalized expression $=$ actual miRNA count/total count of clean reads $\times 10^{6}$.

The $P$-values and fold-change values were examined using normalized data, and the fold-change values used to generate a scatter plot:

Fold-change $=\log _{2}$ (treatment/control).

The $P$-value was determined as follows:

$$
\begin{gathered}
p(x \mid y)=\left(\frac{N_{2}}{N_{1}}\right)^{y} \frac{(x+y) !}{x ! y !\left(1+\frac{N_{2}}{N_{1}}\right)^{(x+y+1)}} \\
C\left(y \leq y_{\text {min }} \mid \mathrm{x}\right)=\sum_{y=0}^{y \leq y_{\text {min }}} p(y \mid \mathrm{x})
\end{gathered}
$$




$$
D\left(y \geq y_{\max } \mid \mathrm{x}\right)=\sum_{y \geq y_{\max }}^{\infty} p(y \mid x)
$$

where $x$ and $y$ denote values of sRNA total clean reads in the control and treatment, respectively, and $N_{1}$ and $\mathrm{N}_{2}$ represent miRNA normalized expression in the library control and treatment, respectively. The Bonferroni method was used to correct $P$-value parallels to differential gene expression ${ }^{48}$.

Prediction of novel miRNAs targets in Brassica campestris. Three software packages were used to analyze and predict mRNA targets of differentially expressed miRNAs including TargetFinder ${ }^{49}$, TAPIR ${ }^{42}$, and psRobot $^{50}$ as described earlier ${ }^{51}$. To achieve a more reliable result and to increase the confidence interval, only binding sites that were predicted by all three software packages were selected.

KEGG prediction of miRNA-related regulatory pathways. Kyoto Encyclopedia of Genes and Genomes (KEGG) pathway (http://www.genome.jp/kegg/pathway.html) database was used to identify the microRNA-related regulatory pathways using a corrected $P$-value $(\leq 0.05)$ with a threshold derived from a hypergeometric test.

Real-time qPCR validation. To confirm the sequencing results, eight miRNAs, whose expression levels represent both up- and down-regulated changes identified in sequencing analysis, were randomly chosen for validation using RT-qPCR as described ${ }^{52}$. The RNA used for RT-qPCR were aliquots of RNA samples used for sequencing and included $0 \mathrm{~h}, 1 \mathrm{~h}, 6 \mathrm{~h}$ and $12 \mathrm{~h}$ RNA of flowering Chinese cabbage. Briefly, $1 \mu \mathrm{g}$ of total RNA was reverse transcribed into cDNA with miRcute miRNA first-strand cDNA synthesis kit (TIANGEN Biotech, Beijing, China). Specific primers were used for qRT-PCR (Table S7). RT-qPCR was performed using LightCyclerR 480 SYBR Green I Master (Roche) following the manufacturer's instructions. U6 served as an internal control. All reactions were carried out using two biological samples with three technological replicates, and novel and conserved miRNAs relative expression level at each time point was calculated using the $2^{-\Delta \Delta C T} \operatorname{method}^{53}$.

Statistical analysis. The statistical analysis used SPSS software (version 22.0; IBM Corp., Armonk, NY, USA) and Student's $t$-test difference between treatments. One-way analysis of variance used Tukey's test for multiple comparisons at the $P<0.05$ or $P<0.01$ significance level. Data were expressed as means \pm SEM.

Received: 7 March 2019; Accepted: 1 October 2019;

Published online: 17 October 2019

\section{References}

1. Chen, J. et al. Development of EST-SSR markers in flowering Chinese cabbage (Brassica campestris L. ssp. chinensis var. utilis Tsen et Lee) based on de novo transcriptomic assemblies. PloS one 12, e0184736 (2017).

2. Kusaba, M., Nishio, T., Satta, Y., Hinata, K. \& Ockendon, D. Striking sequence similarity in inter-and intra-specific comparisons of class I SLG alleles from Brassica oleracea and Brassica campestris: implications for the evolution and recognition mechanism. Proc. Natl. Acad. Sci. USA 94, 7673-7678 (1997).

3. Young, L. W., Wilen, R. W. \& Bonham-Smith, P. C. High temperature stress of Brassica napus during flowering reduces micro-and megagametophyte fertility, induces fruit abortion, and disrupts seed production. J. Exp. Bot. 55, 485-495 (2004).

4. Bita, C. \& Gerats, T. Plant tolerance to high temperature in a changing environment: scientific fundamentals and production of heat stress-tolerant crops. Front. Plant Sci. 4, 273 (2013).

5. Battisti, D. S. \& Naylor, R. L. Historical warnings of future food insecurity with unprecedented seasonal heat. Science 323, 240-244 (2009).

6. Zhai, Z., Lin, Z., Chen, H., Chen, Z. \& Center, G. Temporal and spatial variation of temperature suitability index for Brassica parachinesis in Guangdong. Guang. Agric Sci. 3, 14 (2016).

7. Sato, S., Katoh, N., Iwai, S. \& Hagimori, M. Effect of low temperature pretreatment of buds or inflorescence on isolated microspore culture in Brassica rapa (syn. B. campestris). Breed. Sci. 52, 23-26 (2002).

8. Fan, Z.-Q. et al. BrWRKY65, a WRKY transcription factor, is involved in regulating three leaf senescence-associated genes in Chinese flowering cabbage. Int. J. Mol. Sci. 18, 1228 (2017).

9. LI, R.-H. et al. Effects of Heat Stress on Several Physiological Traits in Heat-yolerant and Heat-Sensitive Genotypes of Flowering Chinese Cabbage [J]. Nort Horti. 1, 000 (2012).

10. Challinor, A., Wheeler, T., Craufurd, P., Ferro, C. \& Stephenson, D. Adaptation of crops to climate change through genotypic responses to mean and extreme temperatures. Agri. Eco Envir. 119, 190-204 (2007).

11. Shukla, L. I., Chinnusamy, V. \& Sunkar, R. The role of microRNAs and other endogenous small RNAs in plant stress responses. Biochimica et Biophysica Acta (BBA)-Gene Regulatory Mechanisms 1779, 743-748 (2008).

12. Khraiwesh, B., Zhu, J.-K. \& Zhu, J. Role of miRNAs and siRNAs in biotic and abiotic stress responses of plants. Biochimica et Biophysica Acta (BBA)-Gene Regulatory Mechanisms 1819, 137-148 (2012).

13. Rogers, K. \& Chen, X. Biogenesis, turnover, and mode of action of plant microRNAs. The Plant Cell 25, 2383-2399 (2013).

14. Piriyapongsa, J. \& Jordan, I. K. Dual coding of siRNAs and miRNAs by plant transposable elements. Rna 14, 814-821 (2008).

15. Sunkar, R. \& Zhu, J.-K. Novel and stress-regulated microRNAs and other small RNAs from Arabidopsis. Plant Cell 16, 2001-2019 (2004).

16. Sieber, P., Wellmer, F., Gheyselinck, J., Riechmann, J. L. \& Meyerowitz, E. M. Redundancy and specialization among plant microRNAs: role of the MIR164 family in developmental robustness. Development 134, 1051-1060 (2007).

17. German, M. A. et al. Global identification of microRNA-target RNA pairs by parallel analysis of RNA ends. Nat.Biotech. 26, 941 (2008).

18. Liu, H. T., Gao, F., Cui, S. J., Han, J. L. \& Zhou, R. G. Primary evidence for involvement of IP 3 in heat-shock signal transduction in Arabidopsis. Cell Res. 16, 394 (2006).

19. Larkindale, J., Hall, J. D., Knight, M. R. \& Vierling, E. Heat stress phenotypes of Arabidopsis mutants implicate multiple signaling pathways in the acquisition of thermotolerance. Plant. physiol. 138, 882-897 (2005). 
20. Pantaleo, V. et al. Identification of grapevine microRNAs and their targets using high-throughput sequencing and degradome analysis. Plant J. 62, 960-976 (2010).

21. Xu, M. Y. et al. Identification of miRNAs and their targets from Brassica napus by high-throughput sequencing and degradome analysis. BMC genomics 13, 421 (2012).

22. Wang, J., Meng, X., Dobrovolskaya, O. B., Orlov, Y. L. \& Chen, M. Non-coding RNAs and Their Roles in Stress Response in Plants. Genom. proteom.bioinfor.(2017).

23. Zhao, J., He, Q., Chen, G., Wang, L. \& Jin, B. Regulation of non-coding RNAs in heat stress responses of plants. Front. Plant Sci. 7, $1213(2016)$.

24. Sailaja, B. et al. Prediction and expression analysis of miRNAs associated with heat stress in Oryza sativa. Rice Sci. 21, 3-12 (2014).

25. Wang, W., Vinocur, B., Shoseyov, O. \& Altman, A. Role of plant heat-shock proteins and molecular chaperones in the abiotic stress response. Trend. plant sci. 9, 244-252 (2004).

26. Contreras-Cubas, C., Palomar, M., Arteaga-Vázquez, M., Reyes, J. L. \& Covarrubias, A. A. Non-coding RNAs in the plant response to abiotic stress. Planta 236, 943-958 (2012).

27. Sunkar, R., Li, Y.-F. \& Jagadeeswaran, G. Functions of microRNAs in plant stress responses. Trend. plant sci. 17, 196-203 (2012).

28. Urano, K., Kurihara, Y., Seki, M. \& Shinozaki, K. 'Omics' analyses of regulatory networks in plant abiotic stress responses. Curr. opin. plant biol. 13, 132-138 (2010).

29. Körbes, A. P. et al. Identifying conserved and novel microRNAs in developing seeds of Brassica napus using deep sequencing. PloS one 7, e50663 (2012).

30. Zhang, J. et al. Genome-wide identification, putative functionality and interactions between lncRNAs and miRNAs in Brassica species. Sci. reports 8, 4960 (2018).

31. Srivastava, A. K., Sablok, G., Hackenberg, M., Deshpande, U. \& Suprasanna, P. Thiourea priming enhances salt tolerance through co-ordinated regulation of microRNAs and hormones in Brassica juncea. Sci. reports 7, 45490 (2017).

32. Jiang, J., Lv, M., Liang, Y., Ma, Z. \& Cao, J. Identification of novel and conserved miRNAs involved in pollen development in Brassica campestris ssp. chinensi s by high-throughput sequencing and degradome analysis. BMC genomics 15, 146 (2014).

33. Yu, X. et al. Identification of conserved and novel microRNAs that are responsive to heat stress in Brassica rapa. J. Exp. Bot. 63, 1025-1038 (2011).

34. Wang, F. et al. High-throughput sequencing discovery of conserved and novel microRNAs in Chinese cabbage (Brassica rapa L. ssp. pekinensis). Mol. genet. genom. 287, 555-563 (2012).

35. Bhardwaj, A. R. et al. A genome-wide perspective of miRNAome in response to high temperature, salinity and drought stresses in Brassica juncea (Czern) L. PloS one 9, e92456 (2014).

36. Chen, C.-C. et al. Comparative miRNAs analysis of Two contrasting broccoli inbred lines with divergent head-forming capacity under temperature stress. BMC genomics 16, 1026 (2015).

37. Sunkar, R. \& Jagadeeswaran, G. In silico identification of conserved microRNAs in large number of diverse plant species. BMC plant biol. 8, 37 (2008).

38. Nozawa, M., Miura, S. \& Nei, M. Origins and evolution of microRNA genes in plant species. Gen. biol. evol. 4, 230-239 (2012).

39. Lukasik, A., Pietrykowska, H., Paczek, L., Szweykowska-Kulinska, Z. \& Zielenkiewicz, P. High-throughput sequencing identification of novel and conserved miRNAs in the Brassica oleracea leaves. BMC genomics 14, 801 (2013).

40. Wang, J. et al. Identification and characterization of microRNAs and their target genes in Brassica oleracea. Gene 505, 300-308 (2012).

41. Li, G. et al. Differentially Expressed microRNAs and Target Genes Associated with Plastic Internode Elongation in Alternanthera philoxeroides in Contrasting Hydrological Habitats. Front. Plant Sci. 8, https://doi.org/10.3389/fpls.2017.02078 (2017).

42. Bonnet, E., He, Y., Billiau, K. \& Van de Peer, Y. TAPIR, a web server for the prediction of plant microRNA targets, including target mimics. Bioinformatics 26, 1566-1568 (2010).

43. Xie, F. \& Zhang, B. Target-align: a tool for plant microRNA target identification. Bioinformatics 26, 3002-3003 (2010).

44. Dai, X. \& Zhao, P. X. psRNATarget: a plant small RNA target analysis server. Nucleic acids res. 39, W155-W159 (2011).

45. Wang, Z., Qiao, Y., Zhang, J., Shi, W. \& Zhang, J. Genome wide identification of microRNAs involved in fatty acid and lipid metabolism of Brassica napus by small RNA and degradome sequencing. Gene 619, 61-70 (2017).

46. Langmead, B. \& Salzberg, S. L. Fast gapped-read alignment with Bowtie 2. Nat. methods 9, 357 (2012).

47. Kozomara, A. \& Griffiths-Jones, S. miRBase: annotating high confidence microRNAs using deep sequencing data. Nucl. Acids Res. 42, D68-D73 (2013).

48. Abdi, H. Bonferroni and Šidák corrections for multiple comparisons. Enc. measur. stat. 3, 103-107 (2007).

49. Xie, F., Xiao, P., Chen, D., Xu, L. \& Zhang, B. miRDeepFinder: a miRNA analysis tool for deep sequencing of plant small RNAs. Plant mol. biol. 80, 75-84 (2012).

50. Wu, H.-J., Ma, Y.-K., Chen, T., Wang, M. \& Wang, X.-J. PsRobot: a web-based plant small RNA meta-analysis toolbox. Nucl. Acids Res. 40, W22-W28 (2012).

51. Srivastava, P. K., Moturu, T. R., Pandey, P., Baldwin, I. T. \& Pandey, S. P. A comparison of performance of plant miRNA target prediction tools and the characterization of features for genome-wide target prediction. Bmc Genomics 15, 348 (2014).

52. Guo, P. et al. Differentially expressed genes between drought-tolerant and drought-sensitive barley genotypes in response to drought stress during the reproductive stage. J. Exp. Bot. 60, 3531-3544 (2009).

53. Livak, K. J. \& Schmittgen, T. D. Analysis of relative gene expression data using real-time quantitative PCR and the $2^{-\Delta \Delta C T}$ method. methods 25, 402-408 (2001).

54. Kanehisa, M. \& Goto, S. KEGG: kyoto encyclopedia of genes and genomes. Nucl. Acids Res. 28, 27-30 (2000).

\section{Acknowledgements}

This work was funded by the Provincial Key International Cooperative Research Platform and the Major Scientific Research Project of Guangdong Higher Education (2015KGJHZ015), and the Science and Technology Plan of Guangdong of China (2016B020201001).

\section{Author contributions}

Conceived and designed the experiments: P.G. Performed the experiments and analyzed data: W.A., Y.X., R.L. and H.Z. Contributed reagents/materials/analysis tools: Y.X., W.A., R.L. and H.Z. Wrote the paper: W.A., G.B., K.S. and P.G.

\section{Competing interests}

The authors declare no competing interests. 


\section{Additional information}

Supplementary information is available for this paper at https://doi.org/10.1038/s41598-019-51443-y.

Correspondence and requests for materials should be addressed to P.G.

Reprints and permissions information is available at www.nature.com/reprints.

Publisher's note Springer Nature remains neutral with regard to jurisdictional claims in published maps and institutional affiliations.

(c) (i) Open Access This article is licensed under a Creative Commons Attribution 4.0 International License, which permits use, sharing, adaptation, distribution and reproduction in any medium or format, as long as you give appropriate credit to the original author(s) and the source, provide a link to the Creative Commons license, and indicate if changes were made. The images or other third party material in this article are included in the article's Creative Commons license, unless indicated otherwise in a credit line to the material. If material is not included in the article's Creative Commons license and your intended use is not permitted by statutory regulation or exceeds the permitted use, you will need to obtain permission directly from the copyright holder. To view a copy of this license, visit http://creativecommons.org/licenses/by/4.0/.

(c) The Author(s) 2019 